Mens

revue d'histoire intellectuelle de l'Amérique française

\title{
Alan Gordon. Making Public Pasts. The Contested Terrain of Montreal's Public Memories, 1891-1930. Montréal et Kingston, McGill-Queen's University Press, 2001. 233 p.
}

\section{Michel Ducharme}

Volume 3, numéro 1, automne 2002

URI : https://id.erudit.org/iderudit/1024622ar

DOI : https://doi.org/10.7202/1024622ar

Aller au sommaire du numéro

Éditeur(s)

Centre de recherche en civilisation canadienne-française

ISSN

1492-8647 (imprimé)

1927-9299 (numérique)

Découvrir la revue

Citer ce compte rendu

Ducharme, M. (2002). Compte rendu de [Alan Gordon. Making Public Pasts. The Contested Terrain of Montreal's Public Memories, 1891-1930. Montréal et

Kingston, McGill-Queen's University Press, 2001. 233 p.] Mens, 3(1), 107-111.

https://doi.org/10.7202/1024622ar 
Morton, de "comporter un fort utile message politique et moral » (Morton, loc. cit., p. 109).

\section{Sean Mills \\ Département d'bistoire \\ Université McGill}

* J'aimerais remercier Benoît Dubrewil pour la traduction ainsi qu'Annie Gagnon et les membres du comité de rédaction de Mens pour leurs précieux commentaires.

\section{Alan Gordon. Making Public Pasts. The Contested Terrain of Montreal's Public Memories, 1891-1930. Montréal et Kingston, McGill-Queen's University Press, 2001. 233 p.}

Avec la récente publication de son ouvrage Making Public Pasts, Alan Gordon ajoute sa contribution à l'histoire culturelle portant sur la commémoration et la représentation du passé au Canada. Il rejoint ainsi les H.V. Nelles, Jonathan Vance, Cecilia Morgan, Colin Coates, Patrice Groulx et autres qui ont donné une vigueur toute nouvelle à ce champ historiographique depuis quelques années. Dans son volume, Gordon aborde les différentes conceptions de l'histoire qui se sont affrontées dans la définition de la mémoire publique à Montréal entre 1891 et 1930 par l'étude des plaques et des monuments commémoratifs. Plus fondamentalement, il traite de la formation et de la transformation des identités collectives qui se sont retrouvées en compétition dans ce qui était la métropole canadienne au tournant du XXe siècle ainsi que du rôle central joué par les petite et moyenne bourgeoisies dans le processus commémoratif. Dans cette perspective, si les pla- 
ques et les monuments érigés à Montréal servent officiellement de point de départ à l'analyse, ils figurent dans les faits principalement comme point d'arrivée à une réflexion identitaire qui les dépasse largement. Ils servent davantage de preuves visant à consolider l'argumentation de l'auteur que de sources à partir desquelles la réflexion est menée. En fait, les monuments semblent assez fréquemment facultatifs dans la démonstration.

Il faut d'emblée reconnaitre qu'étant donné les objectifs de l'auteur, le choix de traiter de Montréal apparaît des plus judicieux. D'abord, nous y retrouvons les tiraillements habituels entre la vision de l'élite (et parfois les visions des élites) et celle de la population. Ensuite, l'étude de la construction de la mémoire publique à Montréal permet de mettre en lumière le clivage existant entre les communautés anglophone et francophone. Si Gordon fait bien ressortir la compétition entre les deux groupes linguistiques pour le contrôle de la mémoire publique, il raffine suffisamment l'analyse pour éclairer certaines tensions existant au sein de chaque communauté. Enfin, l'auteur a choisi un moment critique de l'histoire de Montréal en ce qui concerne l'évolution de l'influence respective des groupes en présence.

Les premiers chapitres constituent une mise en contexte de Montréal et des principaux groupes engagés dans la lutte pour le contrôle de la mémoire publique. Ainsi, les chapitres 2 et 3 font un survol de l'histoire de Montréal des origines à 1930. Quant au chapitre 4, il présente les cinq groupes responsables de l'érection des plaques et des monuments à l'époque étudiée. Bien qu'il s'agisse d'un simple survol, certaines nuances auraient été souhaitables. Par exemple, ces chapitres ne mentionnent ni le désintérêt croissant de la communauté anglophone pour les affaires municipales de Montréal, ni son repli dans les banlieues voisines. Il n'y a en outre aucune réfé- 
rence à l'annexion forcée de la ville de Maisonneuve à Montréal en 1918. Or, cette annexion décidée par le gouvernement du Québec est essentielle dans l'histoire métropolitaine puisqu'elle impose à la ville une dette énorme dont elle n'est pas responsable. Il s'agit là d'un détail important qui relativise l'affirmation voulant que la seule " mauvaise gestion » des élus soit responsable de la précarité de la situation budgétaire de Montréal en 1918. De surcroît, Gordon interprète l'énoncé de lord Durham, voulant que les Canadiens français forment " un peuple sans histoire et sans littérature ", comme une critique du manque d'héritage canadien-français. En fait, remise en contexte, l'accusation signifiait exactement le contraire. Durham reprochait aux Canadiens français de conserver un lourd héritage culturel qui nuisait à leur évolution vers la liberté. Malgré tout, ces chapitres présentent un survol suffisant dans l'ensemble pour permettre au lecteur de suivre la démonstration subséquente.

Avec le chapitre 5 commence vraiment la réflexion sur la mémoire publique. Gordon aborde d'abord la mémoire britannique de Montréal avec son impérialisme, sa loyauté envers le souverain et sa conviction d'adhérer à des valeurs universelles. À ce sujet, il ne fait que reprendre la thèse de Carl Berger sur l'impérialisme anglo-canadien d'avant la Première Guerre mondiale en prenant l'élite anglo-montréalaise comme exemple. Gordon relate également le développement du nationalisme canadien à la suite de la Grande Guerre. Bien que les francophones aient parfois été intégrés dans la conception impérialiste de l'histoire, le patriotisme anglo-saxon montréalais n'a pu établir son hégémonie sur la mémoire publique et a dû composer avec la contestation des francophones de la ville. Le chapitre 6 traite de la mémoire publique canadienne-française. Cette mémoire est d'abord caractérisée, selon Gordon, par la prégnance de la religion catholique. 
Dans la décennie 1920, la mémoire canadienne-française a, en outre, réussi à récupérer les Patriotes. La convergence entre le catholicisme et la mémoire des Patriotes se matérialise par l'inauguration du monument dédié aux Patriotes en 1926.

Les deux derniers chapitres complètent le chapitre théorique sur la mémoire (chapitre 1) et constituent le cœur et le nœud de l'ouvrage. Le chapitre 7 traite de manière assez habile de la concurrence entre les mémoires canadienne-anglaise et canadienne-française. L'auteur adopte alors une conception de l'espace qui intègre la temporalité. Ainsi étudie-t-il la territorialité de la mémoire publique. Enfin, il n'omet pas de mentionner les exclus de cette mémoire: les femmes, les ouvriers et les Amérindiens. Dans le chapitre 8, Gordon se concentre sur les festivals, les défilés et «l'invention » de traditions. Il démontre que les masses proposaient leurs visions du passé à travers les festivals à la fin du XIX $\mathrm{X}^{\mathrm{e}}$ siècle. Ces événements se sont progressivement commercialisés. Gordon soutient que, loin de leur nuire, la commercialisation et la création de nouvelles «traditions » ont permis de sauver ces activités de la disparition. Toutefois, le prix de leur survie fut leur récupération par les élites. Ainsi, de spontanés et de populaires qu'ils étaient, ces festivals sont devenus organisés et ont été soumis à des impératifs idéologiques précis. L'auteur termine sa démonstration en abordant l'évolution de la SaintJean-Baptiste. Il devient clair alors que la spontanéité initiale de l'événement disparaît peu à peu pour faire place à une récupération idéologique de la part de l'élite nationaliste.

D’une manière générale, l'ouvrage ne déborde pas d'originalité et les chapitres sont d'une valeur inégale. Toutefois, il possède quelques qualités importantes. Premièrement, il s'agit d'un ouvrage sérieux portant sur le Québec publié au Canada anglais. Deuxièmement, reconnaissons l'ambition d'un projet qui englobe deux groupes culturels distincts. En effet, 
l'auteur traite aussi bien des anglophones que des francophones, même si le traitement du cas anglophone disparaît progressivement dans les derniers chapitres. Il s'agit d'un choix intéressant parce qu'il réintègre la minorité anglophone montréalaise, trop longtemps négligée, dans l'histoire du Québec. Troisièmement, la réflexion menée par Gordon sur la complexité du lien unissant nation et mémoire vaut la peine d'être lue. Enfin, l'ouvrage éclaire la petite histoire des monuments montréalais. Bref, Gordon nous présente une œuvre qui intéressera les amateurs de l'histoire canadienne en général, québécoise et montréalaise en particulier.

Michel Ducharme

Département d'bistoire

Université McGill

\section{Edmond Robillard. Mémoires d'une enfance à Le Gardeur. Montréal, Edmond Robillard Éditeur, 2001. $331 \mathrm{p}$.}

Edmond Robillard n'écrit pas pour les tièdes. Il nous le rappelle agréablement avec cet essai autobiographique exceptionnel (son $22^{\mathrm{e}}$ livre) dans lequel, en plus de nous faire partager ses souvenirs d'enfance avec une franchise étonnante, il nous livre ses vues pénétrantes sur divers problèmes contemporains tels que le suicide, l'avortement et la décrépitude du système d'éducation. Cet écrivain à la plume ardente, dont on ne finit plus d'admirer les talents de poète, théologien, philosophe, dramaturge et traducteur, s'est beaucoup amusé, de toute évidence, en racontant ses Mémoires, qui s'étendent de sa naissance, en 1917, à sa profession solennelle chez les 